\title{
Características y difusión de las revistas científico-técnicas españolas de ciencias de la actividad física y el deporte
}

\author{
Por Miguel Villamón-Herrera, José Devís-Devís, Alexandra Valencia-Peris y Javier Valenciano-Valcárcel
}

\begin{abstract}
Resumen: El objetivo de este artículo es presentar la situación actual de las revistas españolas de Ciencias de la Actividad Física y el Deporte (CCAFD). Para ello se actualiza el inventario de publicaciones periódicas vigentes y se analizan varias de sus características editoriales básicas como la antigüedad, periodicidad, lugar de edición, institución editora, tipo de soporte y tipo de formato editorial. También se estudia la circulación de dichas publicaciones en las bases de datos nacionales e internacionales selectivas y la difusión que tienen por internet a través de la accesibilidad, el contenido y los servicios añadidos que ofrecen. Se concluye que las CCAFD es un campo joven y poco consolidado académicamente, según indican las características editoriales básicas de sus revistas científico-técnicas. Sin embargo, dichas publicaciones han experimentado una importante mejora en los últimos años en su indización en bases de datos y en su presencia en internet.
\end{abstract}

Palabras clave: Ciencias de la actividad física y el deporte; Revistas españolas; Características editoriales; Bases de datos; Circulación; Visibilidad; Internet.

Title: Dissemination and characteristics of Spanish physical activity and sport sciences scientific and technical journals

Abstract: The aim is to present the current situation of Physical Activity and Sport Sciences (PASS) journals in Spain. The inventory of Spanish journals is updated and several basic editorial characteristics, such as longevity, periodicity, place of publication, editorial institution and support, and type of editorial format are analyzed. The journals' circulation in national and international databases and internet dissemination (based on accessibility, content and added services) are also studied. We conclude that PASS is still a young field that has not yet consolidated its academic presence, as shown by the basic editorial characteristics of it scientific and technical journals. Nonetheless, these journals have seen a significant improvement in their database indexing and internet presence in recent years.

Keywords: Physical activity and sport sciences; Spanish journals; Editorial characteristics; Database; Circulation; Visibility; Internet.

Villamón-Herrera, Miguel; Devís-Devís, José; Valencia-Peris, Alexandra; Valenciano-Valcárcel, Javier. "Características y difusión de las revistas científico-técnicas españolas de ciencias de la actividad física y el deporte". En: El profesional de la información, 2007, noviembre-diciembre, v. 16, n. 6, pp. 605-615.

DOI: 10.3145/epi.2007.nov.07

\section{Introducción}

Las revistas científicas son fundamentales en el ciclo de la ciencia por el papel que desempeñan en la divulgación de los resultados de investigación y porque son el canal de comunicación más utilizado y recono-

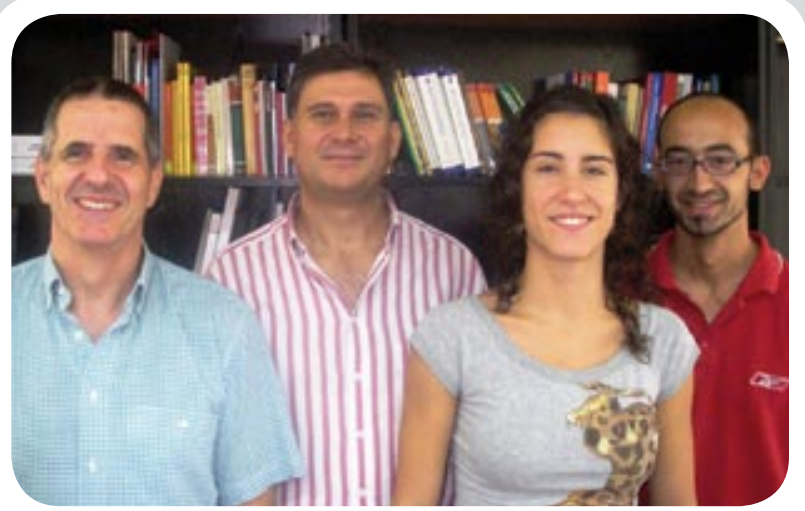

Miguel Villamón es doctor en Educación Física y profesor del Departamento de Educación Física y Deportiva de la Universitat de València, donde imparte docencia desde 1991. Sus líneas de investigación se centran en la evaluación de las publicaciones científicas y la enseñanza de los deportes, temas sobre los que es autor de diversos artículos y monografías.

José Devís es doctor en Ciencias de la Educación y profesor Titular de Universidad en la Facultad de Ciencias de la Actividad Física y el Deporte de la Universitat de València. Sus líneas de investigación son la evaluación de la ciencia, y la pedagogía de la actividad física y la salud. Ha coordinado el libro 'La educación física, el deporte y la salud en el siglo XXI' y es autor de, 'Educación física, deporte y currículum'. Forma parte del consejo editorial de las revistas Sport, Education and society, Physical education and sport pedagogy, y Movimento.

Alexandra Valencia es estudiante de Ciencias de la Actividad Física y el Deporte y Magisterio, becaria-colaboradora de la Unidad de Investigación de Teoría y Pedagogía de la Actividad Física y el Deporte de la Universitat de València.

Javier Valenciano es licenciado en Educación Física y Primer Premio nacional fin de carrera. Ha sido becario FPI del Departamento de Educación Física y Deportiva de la Universitat de València y se ha dedicado al estudio de la calidad y de la producción científica de las revistas españolas de ciencias de la actividad física y el deporte, temas sobre los que ha publicado varios artículos. cido por la comunidad científica. Además resultan primordiales para el desarrollo científico de cualquier campo de conocimiento, siendo su número y calidad indicadores de la madurez del mismo. También sirven para dinamizar a la comunidad académica y se convi- 
erten en elementos de referencia para la evaluación de la actividad investigadora de sus miembros.

En el caso de las Ciencias de la Actividad Física y el Deporte (CCAFD) se observa una mayor presencia de publicaciones periódicas en los circuitos internacionales. Desde hace varias décadas las revistas son indizadas por prestigiosas bases de datos (bdds) internacionales, y un grupo considerable de ellas se recogen en el Journal citation reports (ver Hopkins, 2004, 2005, 2007). Incluso han ido emergiendo bdds específicas como son Spolit, Sport discus y Heracles (Aquesolo, 2000). Sin embargo tienen aún poca visibilidad las revistas españolas y sus trabajos, a pesar del aumento de prestigio académico del mencionado campo en nuestro país. Al menos, no es comparable al aumento de productividad científica y a los recursos que las agencias nacionales y autonómicas han concedido a los investigadores desde la década de 1990 en que se consolidó el estatus académico y universitario de las CCAFD.

\section{"A pesar del aumento de la productividad científica en las CCAFD, las revistas españolas del campo tienen poca visibilidad"}

La falta de estudios sobre las publicaciones periódicas de las CCAFD, a excepción de unos primeros trabajos (Devís, Antolín, Villamón, Moreno y Valenciano, 2003; Devís, Villamón, Antolín, Valenciano y Moreno, 2004; Villamón, Devís y Valenciano, 2005; Villamón, Devís y Valenciano, 2006), no permite encarar las estrategias de mejora necesarias que ayuden a cambiar la situación anterior. Además, esos trabajos necesitan ser actualizados y completados para cumplir satisfactoriamente con dicha tarea. Por ello resulta fundamental abordar el análisis de las revistas científico-técnicas de CCAFD y, de esta manera, conocer en profundidad la situación actual de sus principales órganos de comunicación científica. Concretamente, en este trabajo se analizan las características editoriales básicas y la difusión, en particular la presencia en bdds y el contenido ofrecido en internet de las revistas vigentes en España. La rápida evolución de las publicaciones académicas, debido fundamentalmente al avance tecnológico, y la gran variedad de soportes documentales que existen actualmente (Osca; Mateo, 2003) así lo aconsejan. Pensemos, por ejemplo, en la repercusión editorial que implica la inmediatez electrónica y la posibilidad de acceso universal a los contenidos científicos que representa la iniciativa open access, sin restricciones económicas y de tiempo. Además, en última instancia la información proporcionada por este estudio resulta muy necesaria para todos los agentes participantes, directa o indirectamente, en los distintos momentos del proceso de edición de publicaciones periódicas. Es decir, desde los autores que podrán seleccionar informadamente la revista que mejor se adecue a sus necesidades, hasta los editores que pueden obtener información relevante para mejorar sus productos editoriales o los responsables de la política científica encargada de ayudar al mantenimiento y la internacionalización de las revistas científicas españolas.

\section{Métodos}

Para conocer la situación de las revistas españolas de CCAFD se requiere disponer de un listado de las que están vigentes. Por este motivo, el primer requisito metodológico consiste en elaborar un inventario reciente. Si bien Devís et al. (2003) hicieron un primer inventario con 26 revistas, la constante dinámica de creación y cese de revistas, así como la conveniencia de presentar la situación más reciente del campo de estudio, aconsejaban ofrecer un nuevo listado actualizado de 2007. Esta tarea se realizó a través de la consulta de las siguientes fuentes de información: bases de datos ISSN, ISOC e IME; catálogos colectivos Rebiun, Catálogo colectivo de publicaciones periódicas de la Biblioteca Nacional de España y C17 del Instituto de Salud Carlos III; directorio Latindex; servicio de publicaciones Dialnet; buscadores Google y AltaVista y metabuscador MetaCrawler (que incluye los buscadores Google, Yahoo!, MSN, Ask Jeeves, About, MIVA y LookSmart). También se emplearon los mismos criterios de selección de actualizaciones anteriores (disponer de ISSN, estar activa al menos un año, tener carácter científicotécnico y ajustarse a la temática del campo).

\section{"La existencia de un elevado número de revistas (32 en 2007) disminuye la competencia entre los originales que aspiran a publicarse, lo que puede abrir las puertas a trabajos de menor calidad"}

Una vez conocidas las revistas objeto de estudio, se recogieron los datos relativos a las características editoriales y la difusión de las revistas vigentes. En el caso de las editadas en papel se consultaron directamente los últimos fascículos disponibles en las bibliotecas de la Universitat de València a finales de junio de 2007. Para las revistas electrónicas se analizaron los ejemplares digitales presentes en su web entre los meses de abril y junio del mismo año. En los casos en que fue necesario, 
estos procedimientos se ampliaron a la consulta de las bases de datos bibliográficas mencionadas, a internet y a expertos.

De cada una de las publicaciones se comprobó una serie de datos (título, ISSN, fecha de inicio, lugar de edición, editorial, periodicidad, tipo de soporte, circulación en bases de datos y grado de difusión en internet), de acuerdo con otros trabajos recientes sobre este tema (Abad, González, Martínez, 2005; Gómez del Pulgar, 2006).

\section{Resultados y discusión}

Este apartado está organizado conforme a los dos objetivos del trabajo, las características editoriales básicas y la difusión de las revistas científico-técnicas españolas de CCAFD.

\section{Características editoriales básicas}

La actualización del inventario de publicaciones periódicas científico-técnicas españolas de CCAFD ha proporcionado un listado de 32 revistas vigentes en 2007, que se muestran en la tabla 1 . Se trata de un número similar al de otros campos en España como, por ejemplo, antropología que posee 29 publicaciones, urbanismo con 35 o las 33 revistas de ciencias agrarias (Román, Vázquez, Urdín, 2002; Urdín, Vázquez, Román, 2003). Sin embargo, esta aparente buena salud del campo de estudio no se corresponde con la tradición universitaria e investigadora que poseen los campos anteriores $\mathrm{y}$, probablemente, tampoco con la cantidad de miembros de la comunidad científica. El mantenimiento de este elevado número de revistas puede acabar afectando negativamente a la calidad de las mismas porque requiere disponer de muchos artículos originales. Además, la competencia entre dichos artículos para llegar a ser publicados es considerablemente menor de lo que exigiría un número inferior de revistas.

\section{"El 56,2\% de las revistas se editan en formato papel y electrónicamente, mientras que el $28,1 \%$ lo hace sólo en soporte papel y el $15,6 \%$ sólo electrónicamente"}

exclusivamente en papel. Si se compara esta distribución con la existente en el primer estudio de las revistas del campo correspondientes al año 2000 (Devís et al., 2003) se advierte que, del 7,7\% de publicaciones editadas en versión exclusivamente electrónica, se ha pasado al $15,6 \%$. Pero donde se observa un cambio muy significativo es en la categoría de 'papel y electrónico', pues en 2000 no había ninguna publicación que simultaneara ambos formatos y en 2007 ascienden hasta al 56,2\%. Asimismo, destaca la transformación de 2 revistas a edición online gratuita que antes de 2007 era de pago y editadas en soporte papel: Apunts. Medicina del deporte y Retos. En definitiva, por lo que respecta al tipo de soporte, se observa un crecimiento considerable de la edición electrónica, aunque no sea en exclusividad, que se acompaña en muchos casos de la gratuidad de sus artículos o contenidos en $p d f$, como ocurre en otros campos en España e internacionalmente (Vázquez, Urdín, Román, 2003; Aliaga, Suárez, 2002).

Por otra parte se observa un crecimiento de 6 títulos respecto al inventario del año 2000. Han desaparecido 11 por cese, involución de sus contenidos científicos o retraso en su publicación, mientras que otros 12 han pasado a formar parte del actual inventario. Es, por tanto, el reflejo del dinamismo editorial que durante los últimos años parece mostrar un especial interés por las publicaciones periódicas. Así lo indica también el hecho de que cerca del $50 \%$ se hayan creado en los últimos 10 años (tabla 1). Sólo 11 publicaciones $(34,4 \%)$ son anteriores a 1991, año en que se iniciaron los cursos de doctorado específicos de CCAFD y se intensificó la actividad investigadora, cuestiones éstas íntimamente ligadas a la creación y desarrollo de revistas científicas. No obstante, el número de altas y bajas en las publicaciones también indica una clara dificultad por su continuidad, lo que exige pensar en estrategias de consolidación o, como también se ha sugerido, de integración de títulos (Aleixandre-Benavent, Valderrama-Zurián, González-Alcaide, 2007; Giménez, Gómez, Vázquez, 2001; Krauskopf, Vera, 1995).

\section{"La antigüedad media de las publicaciones periódicas es de 12,4 años y cerca de la mitad se ha creado en los últimos 10 años"}

El tipo de soporte en que se editan estas publicaciones periódicas muestra que sólo en papel existen 9 revistas, otras 18 lo hacen en soporte electrónico y en papel y 5 se editan únicamente en formato electrónico. Esta distribución indica una importante diversificación del soporte de edición que hace tan sólo una década era
En un análisis detallado de la temática se advierte que predominan las revistas multidisciplinares, que suman $18(56,2 \%)$ y poseen un contenido temático diverso relativo a la educación física (4), a las ciencias de la actividad física y el deporte (9), natación y actividades 


\begin{tabular}{|c|c|c|c|c|c|}
\hline Título & ISSN & Inicio & Lugar & Editor & Periodicidad \\
\hline Agua y gestión & $\begin{array}{l}1131- \\
8775\end{array}$ & 1987 & $\begin{array}{l}\text { Esplugues } \\
\text { de Llobregat } \\
\text { (Barcelona) }\end{array}$ & SEAE & Trimestral \\
\hline $\begin{array}{l}\text { Aloma. Revista de psicologia, } \\
\text { ciències de l'educació i de } \\
\text { l'esport }\end{array}$ & $\begin{array}{l}1138- \\
3194\end{array}$ & 1997 & Barcelona & $\begin{array}{l}\text { Blanquerna, Univer- } \\
\text { sitat Ramon Llull }\end{array}$ & Semestral \\
\hline $\begin{array}{l}\text { Apunts. Educación física y } \\
\text { deportes }\end{array}$ & $\begin{array}{l}1577- \\
4015\end{array}$ & $\begin{array}{l}1985 \\
(*)\end{array}$ & Barcelona & $\begin{array}{l}\text { INEFC/Generalitat de } \\
\text { Catalunya }\end{array}$ & Trimestral \\
\hline Apunts. Medicina de l'esport & $\begin{array}{l}0213- \\
3717\end{array}$ & $\begin{array}{l}1985 \\
(*)\end{array}$ & Barcelona & $\begin{array}{l}\text { Societat Catalana de } \\
\text { Medicina de I'Esport } \\
\text { / Consell Català de } \\
\text { I'Esport / Generalitat } \\
\text { de Catalunya }\end{array}$ & Cuatrimestral \\
\hline $\begin{array}{l}\text { Archivos de medicina del } \\
\text { deporte }\end{array}$ & $\begin{array}{l}0212- \\
8799\end{array}$ & 1984 & Pamplona & $\begin{array}{l}\text { Federación Española } \\
\text { de Medicina del De- } \\
\text { porte (Femede) }\end{array}$ & Bimestral \\
\hline $\begin{array}{l}\text { Avances en traumatología, } \\
\text { cirugía ortopédica, rehabili- } \\
\text { tación, medicina preventiva y } \\
\text { deportiva }\end{array}$ & $\begin{array}{l}0214- \\
4077\end{array}$ & 1971 & Barcelona & Asepeyo/Puntex & Trimestral \\
\hline Biomecánica & $\begin{array}{l}1135- \\
2205\end{array}$ & 1992 & Barcelona & $\begin{array}{l}\text { Sociedad Ibérica de } \\
\text { Biomecánica y Bio- } \\
\text { materiales }\end{array}$ & Semestral \\
\hline $\begin{array}{l}\text { Cairon. Revista de ciencias de } \\
\text { la danza }\end{array}$ & $\begin{array}{l}1135- \\
9137\end{array}$ & 1995 & $\begin{array}{l}\text { Alcalá de } \\
\text { Henares } \\
\text { (Madrid) }\end{array}$ & $\begin{array}{l}\text { SP Universidad de } \\
\text { Alcalá }\end{array}$ & $\begin{array}{l}\text { No se indica } \\
\text { (Anual) }\end{array}$ \\
\hline $\begin{array}{l}\text { Comunicaciones técnicas. Pu- } \\
\text { blicación de la ENE de la RFE } \\
\text { de Natación }\end{array}$ & $\begin{array}{l}1135- \\
111 X\end{array}$ & 1988 & Madrid & $\begin{array}{l}\text { Federación Española } \\
\text { de Natación, E.N. } \\
\text { de E. }\end{array}$ & Trimestral \\
\hline $\begin{array}{l}\text { Cuadernos de psicología del } \\
\text { deporte }\end{array}$ & $\begin{array}{l}1578- \\
8423\end{array}$ & 2001 & Murcia & $\begin{array}{l}\text { F. de Psicología, Uni- } \\
\text { versidad de Murcia } \\
\text { / Dirección Gral. de } \\
\text { Deportes de la CARM }\end{array}$ & Semestral \\
\hline Cultura, ciencia y deporte & $\begin{array}{l}1696- \\
5043\end{array}$ & 2004 & Murcia & $\begin{array}{l}\text { Universidad Católica } \\
\text { San Antonio }\end{array}$ & Semestral \\
\hline Derecho deportivo en línea & $\begin{array}{l}1579- \\
2668\end{array}$ & 2001 & Barcelona & $\begin{array}{l}\text { Rafael Alonso Mar- } \\
\text { tínez }\end{array}$ & $\begin{array}{l}\text { No se indica } \\
\text { (Anual) }\end{array}$ \\
\hline Fútbol. Cuadernos técnicos & $\begin{array}{l}1135- \\
2817\end{array}$ & 1995 & Sevilla & $\begin{array}{l}\text { Wanceulen E. Depor- } \\
\text { tiva }\end{array}$ & $\begin{array}{l}\text { No se indica } \\
\text { (Cuatrimestral) }\end{array}$ \\
\hline $\begin{array}{l}\text { Habilidad motriz. Revista de } \\
\text { las ciencias de la actividad } \\
\text { física y el deporte }\end{array}$ & $\begin{array}{l}1132- \\
2462\end{array}$ & 1992 & Córdoba & $\begin{array}{l}\text { Colegio de Licencia- } \\
\text { dos en EF y en CCA- } \\
\text { FD de Andalucía }\end{array}$ & Semestral \\
\hline $\begin{array}{l}\text { Kronos: la revista científica de } \\
\text { la actividad física y el deporte }\end{array}$ & $\begin{array}{l}1579- \\
5225\end{array}$ & 2002 & Madrid & $\begin{array}{l}\text { Universidad Europea } \\
\text { de Madrid }\end{array}$ & Semestral \\
\hline $\begin{array}{l}\text { MD. Revista científica en me- } \\
\text { dicina del deporte }\end{array}$ & $\begin{array}{l}1698- \\
9775\end{array}$ & 2005 & Sevilla & $\begin{array}{l}\text { Junta de Andalu- } \\
\text { cía, Consejería de } \\
\text { Turismo, Comercio } \\
\text { y Deporte, Centro } \\
\text { Andaluz de Medicina } \\
\text { del Deporte }\end{array}$ & $\begin{array}{l}\text { No se indica } \\
\text { (Cuatrimestral) }\end{array}$ \\
\hline
\end{tabular}

Tabla 1. Características editoriales básicas de las revistas científico-técnicas españolas de CCAFD 


\begin{tabular}{|c|c|c|c|c|c|}
\hline Título & ISSN & Inicio & Lugar & Editor & Periodicidad \\
\hline $\begin{array}{l}\text { Motricidad. European journal } \\
\text { of human movement }\end{array}$ & $\begin{array}{l}0214- \\
0071\end{array}$ & 1995 & Cáceres & $\begin{array}{l}\text { Asociación Españo- } \\
\text { la de Ciencias del } \\
\text { Deporte }\end{array}$ & Semestral \\
\hline $\begin{array}{l}\text { NSW. Natación, saltos / sincro } \\
\text { y waterpolo }\end{array}$ & $\begin{array}{l}1136- \\
0003\end{array}$ & 1979 & $\begin{array}{l}\text { Cartagena } \\
\text { (Murcia) }\end{array}$ & $\begin{array}{l}\text { Asociación Españo- } \\
\text { la de Técnicos de } \\
\text { Natación }\end{array}$ & Trimestral \\
\hline RendimientoDeportivo.com & $\begin{array}{l}1578- \\
7354\end{array}$ & 2002 & $\begin{array}{l}\text { Ribaseca } \\
\text { (León) }\end{array}$ & $\begin{array}{l}\text { Juan Carlos Morante } \\
\text { Rábago }\end{array}$ & Cuatrimestral \\
\hline $\begin{array}{l}\text { RED. Revista de entrenamien- } \\
\text { to deportivo }\end{array}$ & $\begin{array}{l}1133- \\
0619\end{array}$ & 1987 & La Coruña & Boidecanto & Trimestral \\
\hline $\begin{array}{l}\text { Retos. Nuevas tendencias en } \\
\text { educación física, deporte y } \\
\text { recreación }\end{array}$ & $\begin{array}{l}1579- \\
1726\end{array}$ & 2002 & Almería & $\begin{array}{l}\text { Federación Española } \\
\text { de Asociaciones de } \\
\text { Docentes de EF }\end{array}$ & Semestral \\
\hline $\begin{array}{l}\text { Revista andaluza de derecho } \\
\text { del deporte }\end{array}$ & $\begin{array}{l}1886- \\
6220\end{array}$ & $\begin{array}{l}2006 \\
(* *)\end{array}$ & Sevilla & $\begin{array}{l}\text { Junta de Andalu- } \\
\text { cía, Consejería de } \\
\text { Turismo, Comercio } \\
\text { y Deporte, Secreta- } \\
\text { ría General para el } \\
\text { Deporte }\end{array}$ & Semestral \\
\hline Revista de educación física & $\begin{array}{l}1133- \\
0546\end{array}$ & 1985 & La Coruña & Boidecanto & Trimestral \\
\hline $\begin{array}{l}\text { Revista de psicología del de- } \\
\text { porte }\end{array}$ & $\begin{array}{l}1132- \\
239 X\end{array}$ & 1992 & $\begin{array}{l}\text { Palma de } \\
\text { Mallorca }\end{array}$ & $\begin{array}{l}\text { Universitat de les } \\
\text { Illes Balears y Uni- } \\
\text { versitat Autònoma } \\
\text { de Barcelona }\end{array}$ & Semestral \\
\hline $\begin{array}{l}\text { Revista española de educación } \\
\text { física y deportes }\end{array}$ & $\begin{array}{l}1133- \\
6366\end{array}$ & $\begin{array}{l}1949 \\
\text { Nueva } \\
\text { etapa: } \\
2004\end{array}$ & Madrid & $\begin{array}{l}\text { CGICO de Licen- } \\
\text { ciados en EF y en } \\
\text { CCAFD }\end{array}$ & Semestral \\
\hline $\begin{array}{l}\text { Revista Iberoamericana de } \\
\text { psicología del ejercicio y del } \\
\text { deporte. Riped. }\end{array}$ & $\begin{array}{l}1886- \\
8576\end{array}$ & 2006 & Sevilla & $\begin{array}{l}\text { Wanceulen E. Depor- } \\
\text { tiva }\end{array}$ & Semestral \\
\hline $\begin{array}{l}\text { Revista iberoamericana de } \\
\text { psicomotricidad y técnicas } \\
\text { corporales }\end{array}$ & $\begin{array}{l}1577- \\
0788\end{array}$ & 2000 & $\begin{array}{l}\text { Las Rozas } \\
\text { (Madrid) }\end{array}$ & $\begin{array}{l}\text { Asociación de Psi- } \\
\text { comotricistas del } \\
\text { Estado Español y } \\
\text { Red Fortaleza de } \\
\text { Psicomotricidad. }\end{array}$ & Trimestral \\
\hline $\begin{array}{l}\text { Revista internacional de cien- } \\
\text { cias del deporte. Ricyde. }\end{array}$ & $\begin{array}{l}1885- \\
3137\end{array}$ & 2005 & Madrid & Ramón Cantó Alcaraz & Trimestral \\
\hline $\begin{array}{l}\text { Revista internacional de medi- } \\
\text { cina y ciencias de la actividad } \\
\text { física y del deporte }\end{array}$ & $\begin{array}{l}1577- \\
0354\end{array}$ & 2000 & Madrid & $\begin{array}{l}\text { C. Virtual CC. De- } \\
\text { porte y Universidad } \\
\text { Autónoma Madrid/ } \\
\text { Vicente Martínez de } \\
\text { Haro }\end{array}$ & Trimestral \\
\hline $\begin{array}{l}\text { Revista jurídica de deporte y } \\
\text { entretenimiento }\end{array}$ & $\begin{array}{l}1575- \\
8923\end{array}$ & 1999 & Pamplona & Aranzadi Editorial & Cuatrimestral \\
\hline $\begin{array}{l}\text { Selección. Revista española } \\
\text { e iberoamericana de medici- } \\
\text { na de la educación física y el } \\
\text { deporte }\end{array}$ & $\begin{array}{l}0214- \\
8927\end{array}$ & 1989 & Madrid & $\begin{array}{l}\text { Federación Españo- } \\
\text { la de Especialistas } \\
\text { en Medicina de la } \\
\text { Educación Física y el } \\
\text { Deporte }\end{array}$ & Trimestral \\
\hline $\begin{array}{l}\text { Tándem. Didáctica de la edu- } \\
\text { cación física }\end{array}$ & $\begin{array}{l}1577- \\
0834\end{array}$ & 2000 & Barcelona & Editorial Graó & Cuatrimestral \\
\hline
\end{tabular}

(*) Es continuación de “Apuntes de medicina deportiva", fundada en 1964. (**) Es continuación de "Derecho deportivo", fundada en 2003.

Tabla 1 (continuación). Características editoriales básicas de las revistas científico-técnicas españolas de CCAFD 
acuáticas (3), fútbol (1) y danza (1). Las otras 14 revistas $(43,8 \%)$ son unidisciplinares. De ellas, 5 corresponden a medicina del deporte, 1 a biomecánica, 3 a derecho deportivo, 1 a didáctica de la educación física, 3 a psicología del deporte y 1 a la psicomotricidad. Entre las revistas del inventario existen 2 que publican de manera muy marginal y casi inexistente contenidos relativos a las CCAFD. Se trata de Aloma. Revista de psicología, ciències de l'educació i de l'esport y Avances en traumatología, Cirugía ortopédica, rehabilitación, medicina preventiva y deportiva. Lo mismo venía sucediendo con la revista Biomecánica durante algunos años, pero desde el 2006 ha incrementado notablemente los contenidos relativos a la actividad física y el deporte.

El análisis de las entidades editoras que figuran en la tabla 1 muestra que las sociedades científicas editan 11 revistas $(34,4 \%)$, las universidades e instituciones públicas $12(37,5 \%)$, en tanto que los editores privados publican otras 12 revistas $(37,5 \%)^{1}$. Su distribución geográfica evidencia una considerable concentración de la actividad editora española, ya que ésta se distribuye entre 11 provincias y en 2 de ellas (Madrid y Barcelona) se aglutina el $50 \%$. La provincia de Sevilla edita 4 revistas (12,5\%), Murcia $3(9,4 \%)$, La Coruña y Navarra 2 revistas cada una, mientras que Baleares, León y Cáceres editan una sola. Esta concentración en Madrid y Barcelona también se aprecia en otros áreas científicas como ciencias sociales y las humanidades (Osca, Mateo, 2003), medicina (Ponce, 2004) y documentación (Delgado, 2001). En cambio, esta tendencia desaparece cuando se analiza exclusivamente la actividad editora de las revistas electrónicas, ya que se observa una dispersión mayor por toda la geografía española (Aguillo, Primo, Vázquez, 2005). Probablemente el menor coste y la facilidad de publicar en soporte electrónico influyan en ello y permitan que instituciones pequeñas y medianas se lancen a la edición digital.

En la figura 1 se muestra la distribución de las revistas por autonomías, con 25 publicaciones (78\%) editadas entre Cataluña, Madrid, Andalucía y Murcia. Este tipo de distribución está próxima a la observada en historia antigua, prehistoria y arqueología, ya que en estas mismas 4 autonomías se publica el $60,6 \%$ de las publicaciones periódicas de dichos campos (García-Marín, Román, 1998). En el conjunto de ciencias sociales y humanidades, el $62 \%$ de los títulos se editan en Madrid, Cataluña y Andalucía (Osca, Mateo, 2003). En el campo particular de la psicología son Madrid, Cataluña y Valencia las autonomías que publican el 70,6\% de las publicaciones periódicas (Osca, Civera, Tortosa, Quiñones, Peñaranda, López, 2005). En todos los estudios mencionados, la Comunidad de Madrid mantie-

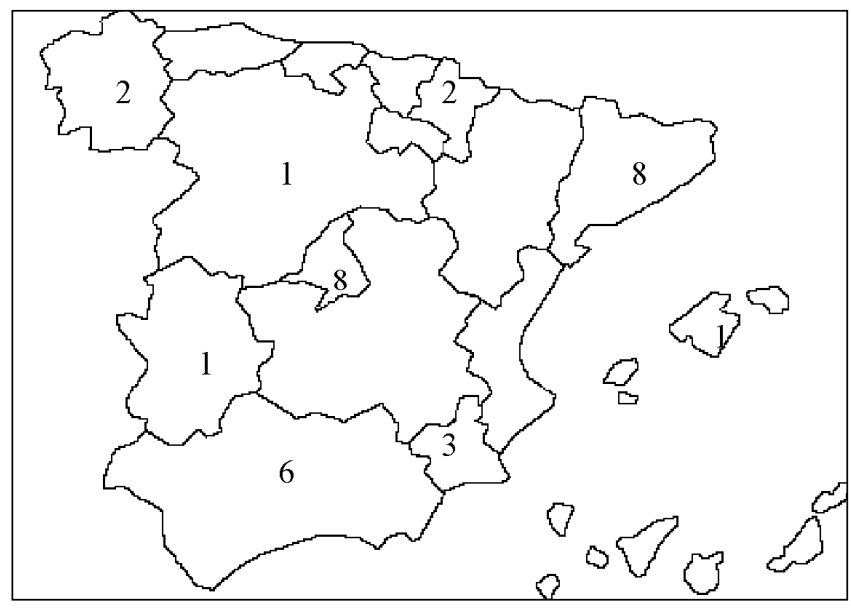

Figura 1. Distribución de la edición de las revistas por Autonomías

ne una actividad editorial destacada, si embargo en una disciplina de las ciencias sociales como es la geografía se observa una mayor concentración de revistas en las regiones periféricas de la península (Olcina, Román, 2004).

\section{"Durante los últimos años ha aumentado la indización de las publicaciones españolas en las bases de datos internacionales, pero todavía existe un amplio margen de mejora"}

La periodicidad más frecuente es la semestral, con 12 revistas $(37,5 \%)$, seguida a escasa distancia por la trimestral con $11(34,4 \%)$. Las cuatrimestrales son 6 $(18,8 \%)$, una sola revista $(3,1 \%)$ tiene periodicidad bimestral y $2(6,3 \%)$ son anuales. No hay ninguna revista que tenga una periodicidad mensual o semanal como ocurre en otros campos más antiguos y desarrollados. Estos datos indican que se trata de un tipo de periodicidad larga, más acorde con la que presentan las publicaciones periódicas de humanidades que con las de ciencia y tecnología o biomedicina. Además, sugieren la existencia de un exceso de publicaciones y/o la falta de artículos originales que, tal y como hemos dicho anteriormente, puede repercutir negativamente en la calidad de las revistas. De acuerdo con Román y Gutiérrez (2005), una revista que publica entre 2 y 4 números por año acredita a la vez un número considerable de suscriptores y una buena calidad de artículos originales. Los autores desean publicar en esa revista, lo que permite a los órganos de dirección proceder a una buena selección de los originales recibidos con el fin de promover la calidad científica de la misma. A la vez, se trata también de un índice de buena salud económica 
o, al menos, de estabilidad financiera. Por el contrario, una publicación anual está casi siempre asociada a una falta de medios para editar de manera más frecuente y/o, también, a la escasez de manuscritos recibidos, lo que impide sacar más de un número por año y limita las posibilidades de selección de originales.

Una atención particular requieren los problemas de puntualidad y cumplimiento de la periodicidad de las revistas españolas de CCAFD. Así se detectan periodos sin cubrir (Retos) o, revistas que optan por editar con posterioridad los números atrasados como si se hubiesen publicado en el año correspondiente (RendimientoDeportivo.com). También se observa la publicación sistemática de números dobles para cumplir, aunque con retraso, la periodicidad propuesta (Cuadernos de psicología del deporte). Otras revistas que desde su comienzo y durante cierto tiempo declaran tener una determinada periodicidad cambian, sin previo aviso, a editar anualmente menos números de los previstos ( $\mathrm{Co}$ municaciones técnicas; Fútbol. Cuadernos técnicos; Tándem. Didáctica de la educación física). Asimismo, algunas revistas acumulan de forma sistemática importantes retrasos en su fecha de salida (Fútbol. Cuadernos técnicos; Habilidad motriz; Revista española de educación física y deportes). Todas estas irregularidades editoriales esconden, muy probablemente, dificultades para conseguir suficiente número de originales y suscriptores o para llevar adelante un proceso eficaz de gestión editorial. En este último caso los problemas suelen derivarse de una deficiente estructura y profesionalización de dichos procesos porque, a menudo, son asumidos por el esfuerzo aislado de un promotor individual que realiza todas las funciones editoriales (Delgado, 2001).

\section{Difusión de las revistas}

La indización en bdds selectivas, es decir, las que exigen a las publicaciones el cumplimiento de diversos criterios de calidad, se muestra en la tabla 2. Se advierte que es muy dispar, pues va desde alguna que tan sólo aparece en algún servicio documental, hasta otras que son registradas por varias bdds de prestigio.

A pesar de la importancia que tiene la indización en bdds selectivas para las propias revistas, pues entre otras ventajas aumenta de forma notable su visibilidad, sólo 11 de las 18 revistas que aparecen en la tabla 2 declaran ser indizadas por alguna bdd y las otras 7 no lo indican. Las 14 revistas restantes del total de publicaciones periódicas de CCAFD no aparecen en ninguno de los servicios documentales consultados. Al comparar estos datos con los de estudios anteriores en el mismo campo, se advierte un considerable incremento en la visibilidad puesto que en el año 2000 había indizadas 4 revistas en bdds $(15,4 \%)$ (Devís et al., 2003), cifra que aumentó a $6(18,8 \%)$ en 2005 (Villamón, Devís,
Valenciano, 2005), y que ha pasado a 18 publicaciones $(56,2 \%)$ indizadas en alguna bdd o servicio documental selectivo en 2007. Este incremento es similar al producido en otros campos en España como, por ejemplo, el de Ciencias de la Salud (Vázquez, Urdín, Román, 2003).

Un análisis más detallado revela que la bdd internacional Heracles/Sportdoc, producida en Francia, es la que más revistas españolas acoge, con 5. Existen 3 bdds (Embase/Excerpta medica, PsycINFO y SIRC/SportDiscus) que indizan cada una 2 revistas españolas. Otras 5 bdds selectivas y de prestigio (Scopus, la biblioteca virtual SciELO, Pascal, CAB Health/CAB Abstracts, EbscoHost) registran cada una de ellas los contenidos de una sola revista. Entre las bdds creadas en América Latina y que ofrecen listados de revistas clasificadas por su calidad, la brasileña Qualis de Capes califica a 4 publicaciones españolas, mientras que la hemeroteca mexicana en línea $\operatorname{RedALyC}$ registra 1 sola.

En cuanto a las bdds españolas, las del CSIC, es decir, ISOC (ciencias sociales) e IME (biomedicina) indizan 13 revistas entre las dos. La bdd Ibecs recoge 3 y, finalmente, Psicodoc registra 2.

Entre las bdds orientadas a ofrecer información que atiende a una serie de indicadores o de índices bibliométricos, a través de listados de revistas, cabe destacar el catálogo Latindex en el que se encuentran registradas 10 publicaciones periódicas del campo. DICE (Difusión y calidad editorial de las revistas españolas de humanidades y ciencias sociales y jurídicas) creada por el Cindoc-CSIC, registra asimismo 10 revistas. La información de esta bdd se complementa con la de otra de la misma institución, se trata de RESH (Valoración integrada e índice de citas de las revistas españolas de ciencias sociales y humanidades), en la que tan solo aparecen 3 revistas. In-Recs (Índice bibliométrico de impacto de las revistas españolas de ciencias sociales), elaborado por el grupo de investigación $E C^{3}$ de la $U n i$ versidad de Granada, registra 5 revistas. Finalmente, otras 2 revistas electrónicas están alojadas en la plataforma digital E-Revistas, dentro del portal Tecnociencia.

La difusión en internet de las publicaciones periódicas españolas de CCAFD ha aumentado de forma destacada en los últimos años. Con la excepción de 3 revistas (Aloma; RED. Revista de entrenamiento deportivo; y Revista de educación física), todas las demás se han podido encontrar directamente a través del citado metabuscador MetaCrawler introduciendo en la casilla de búsqueda sus títulos completos o buscando en la web de los editores de las mismas. Además, al comparar la presencia de las revistas en la web con estudios anteriores, se observa claramente dicho crecimiento ya que del 


\begin{tabular}{|c|c|c|c|}
\hline Título & $\begin{array}{l}\text { Bdds } \\
\text { y servicios extranjeros }\end{array}$ & $\begin{array}{l}\text { Bdds } \\
\text { españolas }\end{array}$ & $\begin{array}{l}\text { Servicios } \\
\text { españoles }\end{array}$ \\
\hline $\begin{array}{l}\text { Aloma. Revista de psicologia, ciències de } \\
\text { l'educació i de l'esport }\end{array}$ & & & In-Recs \\
\hline Apunts. Educación Física y Deportes & $\begin{array}{l}\text { Heracles/Sportdoc } \\
\text { Catálogo Latindex } \\
\text { Qualis (B) }\end{array}$ & ISOC & $\begin{array}{l}\text { In-Recs } \\
\text { DICE } \\
\text { RESH }\end{array}$ \\
\hline Apunts. Medicina de l'esport & Heracles/Sportdoc & & \\
\hline Archivos de medicina del deporte & $\begin{array}{l}\text { Embase/Excerpta Medica } \\
\text { SIRC/SportDiscus } \\
\text { Catálogo Latindex } \\
\text { Qualis (B) }\end{array}$ & $\begin{array}{l}\text { IME } \\
\text { Ibecs }\end{array}$ & \\
\hline Biomecánica & & IME & \\
\hline Cuadernos de psicología del deporte & Catálogo Latindex & ISOC & $\begin{array}{l}\text { In-Recs } \\
\text { DICE }\end{array}$ \\
\hline Cultura, ciencia y deporte & $\begin{array}{l}\text { Catálogo Latindex } \\
\text { Qualis (C) }\end{array}$ & ISOC & DICE \\
\hline $\begin{array}{l}\text { Habilidad motriz. Revista de las ciencias de } \\
\text { la actividad física y el deporte }\end{array}$ & & & $\begin{array}{l}\text { In-Recs } \\
\text { DICE }\end{array}$ \\
\hline $\begin{array}{l}\text { Motricidad. European journal of human } \\
\text { movement }\end{array}$ & Catálogo Latindex & ISOC & DICE \\
\hline RED. Revista de entrenamiento deportivo & & ISOC & \\
\hline $\begin{array}{l}\text { Retos. Nuevas tendencias en educación } \\
\text { física, deporte y recreación }\end{array}$ & Catálogo Latindex & ISOC & $\begin{array}{l}\text { In-Recs } \\
\text { DICE }\end{array}$ \\
\hline Revista de educación física & & ISOC & \\
\hline Revista de psicología del deporte & $\begin{array}{l}\text { PsycINFO } \\
\text { Ebsco Host } \\
\text { SIRC/SportDiscus } \\
\text { SciELO } \\
\text { Heracles/Sportdoc } \\
\text { Catálogo Latindex } \\
\text { Qualis (C) }\end{array}$ & $\begin{array}{l}\text { ISOC-Psic } \\
\text { Ibecs } \\
\text { Psicodoc }\end{array}$ & $\begin{array}{l}\text { In-Recs } \\
\text { DICE } \\
\text { RESH }\end{array}$ \\
\hline $\begin{array}{l}\text { Revista internacional de ciencias del } \\
\text { deporte. Ricyde. }\end{array}$ & $\begin{array}{l}\text { PsycINFO } \\
\text { SportDiscus/SIRC } \\
\text { Sportdoc/ Heracles } \\
\text { Catálogo Latindex } \\
\text { RedALyC }\end{array}$ & & $\begin{array}{l}\text { DICE } \\
\text { E-Revistas }\end{array}$ \\
\hline $\begin{array}{l}\text { Revista internacional de medicina y } \\
\text { ciencias de la actividad física y del deporte }\end{array}$ & $\begin{array}{l}\text { Sportdoc/Heracles } \\
\text { RedALyC (la revista lo } \\
\text { anuncia, pero no aparece) }\end{array}$ & & E-Revistas \\
\hline $\begin{array}{l}\text { Revista jurídica de deporte y } \\
\text { entretenimiento }\end{array}$ & & & DICE \\
\hline $\begin{array}{l}\text { Selección. Revista española e } \\
\text { iberoamericana de medicina de la } \\
\text { educación física y el deporte }\end{array}$ & $\begin{array}{l}\text { Scopus } \\
\text { Embase/Excerpta Medica } \\
\text { Pascal } \\
\text { CAB Health/CAB Abstracts } \\
\text { Catálogo Latindex }\end{array}$ & $\begin{array}{l}\text { IME } \\
\text { Ibecs } \\
\text { Psicodoc }\end{array}$ & \\
\hline Tándem. Didáctica de la educación física & Catálogo Latindex & ISOC & $\begin{array}{l}\text { In-Recs } \\
\text { DICE } \\
\text { RESH }\end{array}$ \\
\hline
\end{tabular}


30,8\% del año 2000 (Devís et al., 2003) se ha pasado al $90,6 \%$ en 2007. Estos últimos datos son similares a los obtenidos por las revistas jurídicas (Gómez del Pulgar, 2006) y las de ciencias de la salud (Vázquez, Urdín, Román, 2003).

Por lo que respecta al tipo de acceso vía internet, se observa que casi la mitad $(46,9 \%)$ ofrece la consulta a través de una web propia e independiente, muy por encima del 3,7\% de las revistas jurídicas españolas (Gómez del Pulgar, 2006). Para el resto se hace a través de un enlace $(37,5 \%)$ y sólo 2 son accesibles consultando el catálogo de publicaciones correspondiente en la web de su editor (ver tabla 3 ).

Quizá el aspecto de más interés para los lectores, en relación con la difusión de las revistas en internet, es el contenido disponible. Es muy variado, y en la tabla 3 se ha intentado categorizarlo a través de siete posibilidades. Así, por ejemplo, 26 revistas ofrecen una referencia básica, aunque en ocasiones es tan pobre que en ella no aparecen datos tan importantes como el ISSN o la periodicidad, tal y como sucede con las revistas Agua y gestión; Cairon; Comunicaciones técnicas y Revista andaluza de derecho del deporte. Un grupo de 17 revistas ofrecen el acceso a todos los sumarios y otras 13 presentan una versión íntegra de sus contenidos (5 de pago y 8 gratuitas). Las publicaciones que ofrecen la versión íntegra en internet suponen un $40,6 \%$, un porcentaje nada despreciable. También existen otras 2 revistas (Avances y Revista española de educación física y deportes) que ofrecen algún número completo escaneado en $p d f$, y sin embargo, paradójica-

\begin{tabular}{|l|l|r|r|}
\hline & & No & \multicolumn{1}{c|}{$\%$} \\
\hline \multirow{4}{*}{$\begin{array}{l}\text { Tipo de } \\
\text { acceso }\end{array}$} & Dirección propia & 15 & 46,9 \\
\cline { 2 - 4 } & Desde web del editor & 12 & 37,5 \\
\cline { 2 - 4 } & Desde catálogo & 2 & 6,2 \\
\hline \multirow{4}{*}{ Contenidos } & Referencia básica & 26 & 81,2 \\
\cline { 2 - 4 } & Último sumario & 19 & 59,3 \\
\cline { 2 - 4 } & Varios sumarios & 3 & 9,3 \\
\cline { 2 - 4 } & Todos los sumarios & 17 & 53,1 \\
\cline { 2 - 4 } & Algún trabajo completo & 6 & 18,7 \\
\cline { 2 - 4 } & Versión íntegra & 13 & 40,6 \\
\cline { 2 - 4 } & $\begin{array}{l}\text { Información } \\
\text { complementaria }\end{array}$ & 16 & 50,0 \\
\hline \multirow{5}{*}{ Servicios } & Suscripción por internet & 9 & 28,1 \\
\cline { 2 - 4 } & $\begin{array}{l}\text { Correo electrónico de } \\
\text { contacto }\end{array}$ & 14 & 43,7 \\
\cline { 2 - 4 } & Buscador & 1 & 3,1 \\
\hline
\end{tabular}

Tabla 3. Tipo de acceso, contenidos y servicios ofertados en internet por las revistas mente, no presentan ninguna página con su información básica.

\section{“El $81,2 \%$ de las revistas están presentes en internet al menos con una referencia básica, algo más de la mitad permite el acceso a todos los sumarios y una cuarta parte ofrece el texto completo gratuito"}

Por último, los servicios que proporcionan a través de internet, un valor añadido a la edición tradicional en papel, se limita a una simple dirección de correo electrónico para contactar con los editores en 14 revistas de CCAFD. La posibilidad de realizar la suscripción mediante un formulario elaborado al efecto lo ofrecen otras 9, mientras que sólo 1 de ellas (Apunts. Medicina de l'esport) incluye un sistema de búsqueda.

\section{Conclusiones}

El estudio sobre las características y difusión de las revistas científico-técnicas españolas de CCAFD ofrece las siguientes conclusiones:

- Existe un total de 32 publicaciones periódicas en el año 2007, 6 títulos más que en el año 2000.

- Las revistas editadas exclusivamente en soporte papel son el $28,1 \%$, frente al $56,2 \%$ que lo hacen en papel y electrónicamente y el 15,6\% sólo electrónicamente.

- El $65,6 \%$ de las revistas se han fundado con posterioridad a 1991 y cerca de la mitad de ellas en los últimos diez años.

- Existen más revistas multidisciplinares $(56,2 \%)$ que unidisciplinares $(43,8 \%)$.

- Las asociaciones científicas y las universidades e instituciones públicas editan más de dos terceras partes de las revistas. Las instituciones privadas, principalmente editoriales comerciales, publican otra tercera parte.

- La mitad de las revistas se editan entre las provincias de Barcelona y Madrid. Las autonomías de Cataluña, Madrid, Andalucía y Murcia editan más del $78 \%$ de las publicaciones.

- Aproximadamente la mitad de las revistas españolas de CCAFD tiene una periodicidad semestral y anual.

- Existen 11 revistas $(34,4 \%)$ que declaran estar indizadas por alguna bdd, aunque otras $7(21,9 \%)$ también lo están y no lo indican. 
- Las bases de datos internacionales de tipo selectivo indizan 12 revistas $(37,5 \%)$. Hay 1 revista que está presente en 7, y 2 en 5 bases de datos.

- El 81,2\% de las revistas está presentes en internet al menos con una referencia básica. Los contenidos que ofrecen son muy variados, destacando que el $53,1 \%$ permite el acceso a todos los sumarios y el $25 \%$ al texto completo gratuito.

Como puede observarse, las características editoriales básicas indican que las CCAFD es un campo joven y poco consolidado académicamente. El número de títulos sigue creciendo, pero la mitad de las revistas son de una periodicidad semestral y anual, lo cual puede indicar demasiadas revistas y/o poca cantidad de artículos originales. Además, el predominio de revistas multidisciplinares indica una baja especialización. Una oferta excesiva de títulos influye negativamente en la calidad de las revistas al dispersarse también el número de artículos originales potenciales, disminuyendo la solidez y posicionamiento internacional de las revistas españolas de CCAFD. La distribución geográfica de la edición es similar al de otras disciplinas de ciencias sociales y humanas. La indización en las bases de datos internacionales ha mejorado en los últimos años, pero podría incrementarse bastante más. Asimismo, se observa una marcada tendencia a la presencia de las revistas en internet, aunque los servicios que ofrecen son todavía muy básicos.

\section{Nota}

1. La suma total de 35 revistas, en lugar de las 32 del inventario, es debida a que en 3 casos la edición es asumida por 2 entidades/actores a la vez.

\section{Reconocimiento}

Agradecemos al Ministerio de Educación la ayuda recibida por el proyecto de investigación SEJ2004-03996/EDUC con la que se ha financiado este trabajo

\section{Bibliografía}

Abad, María-Francisca; González, Aurora; Martínez, Celeste. "Características de las revistas médicas españolas. 2004". En: El profesional de la información, 2005, septiembre-octubre, v. 14, n. 5, pp. 380-390.

Aguillo, Isidro; Primo, Elena; Vázquez, Manuela. "Evaluación de las revistas electrónicas de ciencias de la salud, editadas en España frente a los criterios de calidad del sistema Latindex". En: XI Jornadas de información y documentación en ciencias de la salud, Terrassa, 2005. Consultado en: 13/04/2006.

http://www.jornadasbibliosalud.net/c34.pdf

Aleixandre-Benavent, Rafael; Valderrama-Zurián, Juan-Carlos; González-Alcaide, Gregorio. "El factor de impacto de las revistas científicas: limitaciones e indicadores alternativos". En: El profesional de la información, 2007, enero-febrero, v. 16, n. 1, pp. 4-11.

Aliaga, Francisco M.; Suárez, Jesús. Tendencias actuales en la edición de revistas electrónicas: nueva etapa en Relieve. En: Revista electrónica de investigación y evaluación educativa, 2002, v. 8, n. 1. Consulta en: 7/08/2004.

http://www.uv.es/RELIEVE/v8n1/RELIEVEv8n1_o.htm

Aquesolo, José. "Apuntes para una historia de la documentación deportiva”. En: Revista general de información y documentación, 2000, v. 10, n. 1, pp. 31-67.
Delgado-López-Cózar, Emilio. "Las revistas españolas de ciencias de la documentación: productos manifiestamente mejorables". En: El profesional de la información, 2001, diciembre, v. 10, n. 12, pp. 46-56.

Devís, José; Antolín, Luis; Villamón, Miguel; Moreno, Alberto; Valenciano, Javier. "Las revistas científico-técnicas españolas de las ciencias de la actividad física y el deporte: inventario y análisis de la calidad de contenido y difusión". En: Revista española de documentación científica, 2003, v. 26, n. 2, pp. 177-190.

Devís, José; Villamón, Miguel; Antolín, Luis; Valenciano, Javier; Moreno, Alberto. "Las revistas científico-técnicas españolas de ciencias de la actividad física y el deporte: adecuación a las normas ISO y grado de normalización”. En: Ciência da informação, 2004, v. 33, n. 1, pp. 38-47.

García-Marín, Ángeles; Román, Adelaida. "Las publicaciones periódicas de historia antigua, prehistoria y arqueología: difusión internacional". En: Trabajos de prehistoria, 1998, v. 55, n. 1, pp. 139-146.

Giménez, Elea; Gómez, Isabel; Vázquez, Manuela. "Difusión nacional e internacional de las revistas científicas". En: Román, Adelaida (coord.). La edición de revistas científicas. Guía de buenos usos. Madrid: CINDOC, 2001, pp. 35-46. ISBN 84-00-07916-7.

Gómez-del-Pulgar, Gloria. "Difusión por internet de las revistas jurídicas españolas de edición impresa". En: Revista española de documentación científica, 2006, v. 29, n. 2, pp. 258-285.

Hopkins, Will G. (2004). "Impact factor of journals in sport and exercise science, 2000-2003". Sportscience, n. 8, pp. 12-19. Consulta en: 7/07/2006.

http://sportsci.org/jour/04/wghif.htm

Hopkins, Will G. (2005). "Impact factor of journals in sport and exercise science, 2004”. Sportscience, n. 9, pp. 14-16. Consulta en: 7/07/2006. http://www.sportsci.org/jour/05/wghif.htm

Hopkins, Will G. (2007). "The Tour de Journals 2007: Impact Factors in Exercise and Sport”. Sportscience, n. 11, pp. 9-11. Consulta en: 13/09/2007. http://www.sportsci.org/j2007/wghif.htm

Krauskopf, Manuel; Vera, María-Inés. "Las revistas latinoamericanas de corriente principal: indicadores y estrategias para su consolidación". En: Interciencia, 1995, mayo-junio, v. 20, n. 3, pp. 144-148.

Olcina, Jorge; Román, Adelaida. "Las revistas españolas de geografía: cambios y adaptación a los criterios editoriales de calidad". En: García Ramón, María Dolores (eds.) Proceedings. La geografía española ante los retos de la sociedad actual: aportación española al XXX Congreso de la Unión Geográfica Internacional. Glasgow (UK), 2004, pp. 145-179. Consulta en: 23/03/2005.

http://eprints.rclis.org/archive/00002884/.

Osca, Julia; Mateo, María Elena. "Difusión de las revistas españolas de ciencias sociales y humanidades. Acercamiento bibliométrico". En: Revista general de información y documentación, 2003, v. 13, n. 1, pp. 115-132.

Osca, Julia; Civera, Cristina; Tortosa, Francisco; Quiñones, Elena; Peñaranda, María; López, Juan José. "Difusión de las revistas españolas de psicología en bases de datos nacionales e internacionales". En: Anales de documentación, 2005, n. 8, pp. 165-186.

Ponce, Concepción. Análisis de la circulación de las revistas biomédicas españolas en bases de datos nacionales e internacionales (Tesis doctoral). Valencia: Universitat de València, 2004.

Román, Adelaida; Vázquez, Manuela; Urdín, Carmen. "Los criterios de calidad editorial Latindex en el marco de la evaluación de las revistas españolas de humanidades y ciencias sociales". En: Revista española de documentación científica, 2002, v. 25, n. 3, pp. 286-307.

Román, Adelaida; Gutiérrez, Beatriz. "Étude sur les revues espagnoles en sciences humaines et sociales". En: Minon, Marc; Chartron, Ghislaine (coords.). État des lieux comparatif de l'offre de revues SHS, FranceEspagne-Italie. Rapport pour le Ministère français de la recherche, 2005, juin, pp. 27-46. Consulta en: 2/08/2006. http://archivesic.ccsd.cnrs.fr/ docs/00/06/26/64/PDF/sic_00001561.pdf

Urdín, Carmen; Vázquez, Manuela; Román, Adelaida. "Los criterios de calidad editorial Latindex en el marco de evaluación de las revistas españolas de ciencia y tecnología”. En: Revista española de documentación científica, 2003, v. 26, n. 1, pp. 56-73. 
Vázquez, Manuela; Urdín, Carmen; Román, Adelaida. "Las revistas españolas de ciencias de la salud frente a los criterios de calidad editorial Latindex”. En: Revista española de documentación científica, 2003, v. 26, n. 4 , pp. $418-432$.

Villamón, Miguel; Devís, José; Valenciano, Javier. "Análisis de la visibilidad de las revistas científico-técnicas españolas de ciencias de la actividad física y el deporte”. En: Revista de psicología del deporte, 2005, v. 14, n. 2, pp. 253-267.

Villamón, Miguel; Devís, José; Valenciano, Javier. "Análisis de las 'Instrucciones para autores' de las revistas españolas de ciencias de la actividad física y el deporte". En: Motricidad, 2006, n. 16, pp. 133-150.
Miguel Villamón, José Devís, Alexandra Valencia y Javier Valenciano. Facultad de Ciencias de la Actividad Física y el Deporte, Universitat de València. Departamento de Educación Física y Deportiva. C/ Gascó Oliag, 3. 46010 Valencia.

miguel.villamon@uv.es

jose.devis@uv.es

vapea@alumni.uv.es

francisco.valenciano@uv.es

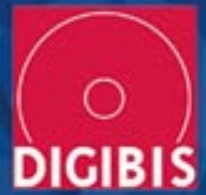

\section{espacios virtuales gestión del}

\section{conocimiento preservación digital}

Herramientas para crear espacios virtuales

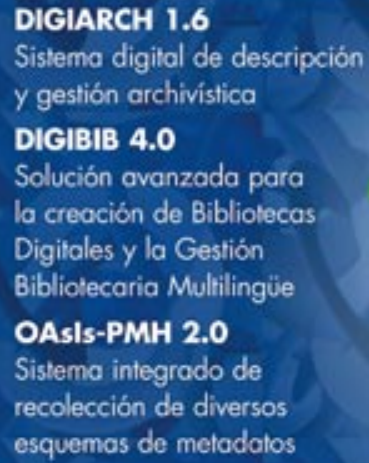

\section{OAsIs-PMH 2.0}

Sistema integrado de recolección de diversos esquemas de metadatos

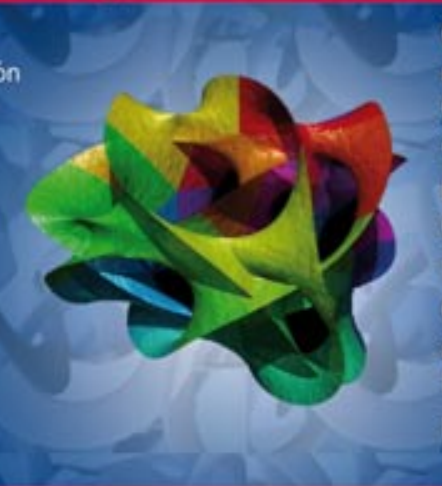

OA (Open Acces) mediante Driver Creación de repositorios OA a partir de $O A$

Digitalización avanzada Con asignación dinámica de metadatos

Consultoria sobre Web semántica y ontologías Elaboración de directrices, programas de trabajo

\section{ORACLE}

- Tecnologías abiertas para la creación, recuperación y recolección de metadatos $y$ anotación de instancias (MARCXML, DCMI y RDF)

- FRBR (IFLA)/CRM (ICOM) ISO 21127:2006

- Recolección en la Web para Entidades e Instituciones de Memoria en OAI-PMH y Dublin Core e intercambio de metadatos en METS (diferentes Profiles)

- Repositorios Institucionales para Preservación Digital a largo plazo mediante PREMIS y OAIS ISO 14721

\section{www.digibis.com}




\section{Applying Web 2.0: Innovation, Impact and Implementation}

\section{4-6 December \\ Olympia Grand Hall, London, UK}

THE NO. 1 CONFERENCE FOR THE INFORMATION WORLD

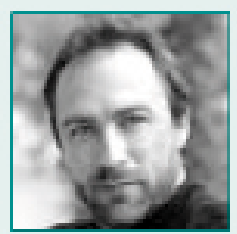

Opening kernote speaker

Jimmy Woles. Founder, Wikipedia \& Wikia

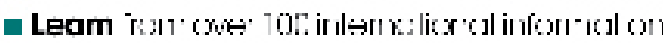

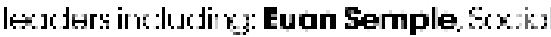
Coma ing Expert Greg Hotess, Marrand Stcte!.. rimersity, Stephen Abram, SirsiDprix ond Gunnar Sahlin, Nojicnoll Lerory of Swoster

- Discaver vitrot wees 20 reecus for put

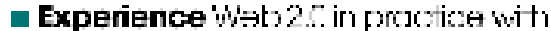

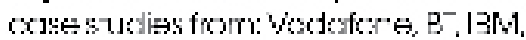
Dr.yjeccese 1.rmersity of S"eff els, Yozills Fircreandroce

- Gain new skilsord comestencies for inferroution pofessional 20

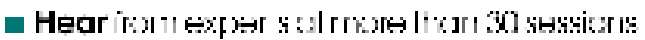

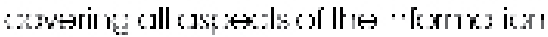

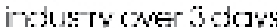

- Nelwork with your paers form coner A5countries:

- Develop wo.. - Web 2.0. Web Search'. 'Research \& Compelitive Intelligence skil: rnd knwwledge in tt' Mncky 3 Decemtar

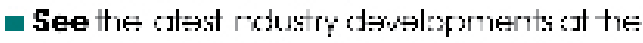
2 co-located free exhibitions of (y,ar 250 online content arrd in orrmotion merrọerrant sold fic"s serwictars

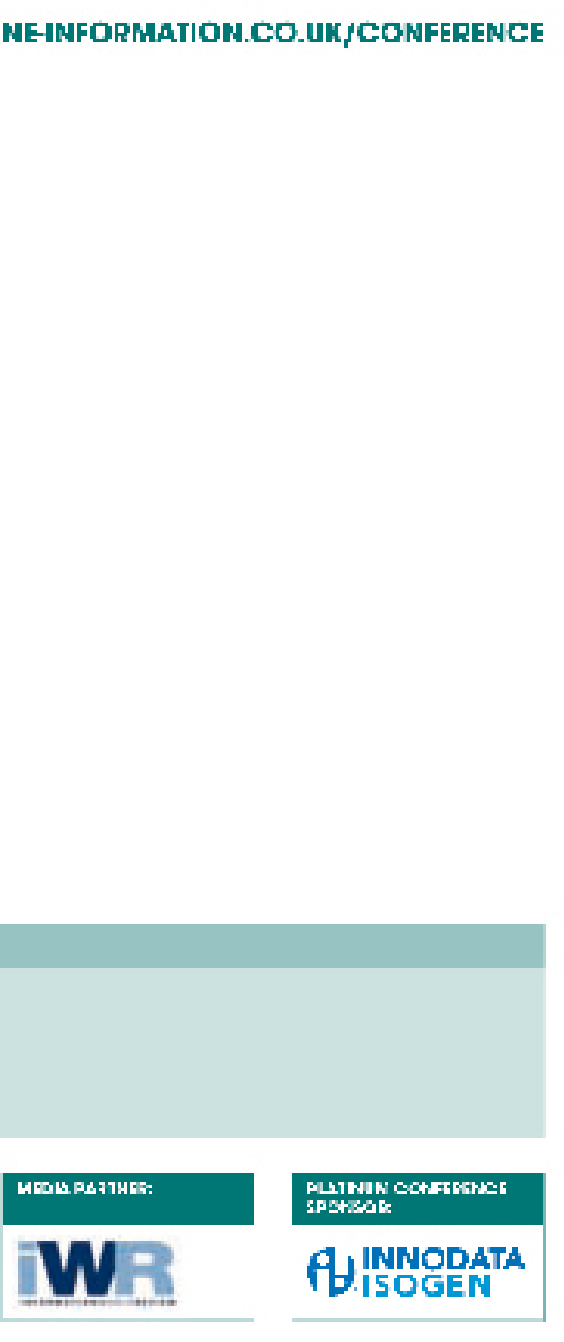

supporiter ay:

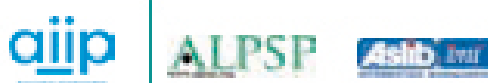
\begin{tabular}{l|ll} 
Cig & $\frac{\text { cilip }}{\text { ensia }}$ \\
\hline dfid & eusidic freepint
\end{tabular}

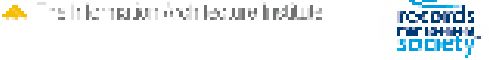

SLA mSPIN WKGIC

www.online-information.co.uk/conference 